\title{
Effect of Surface Anisotropy in Core-Shell Bimetallic Nanoparticles
}

\author{
E.E. Tornau ${ }^{a, *}$, V. Petrauskas ${ }^{a}$ And O. Crisan $^{b}$ \\ ${ }^{a}$ Semiconductor Physics Institute, Goštauto 11, 01108 Vilnius, Lithuania \\ ${ }^{b}$ Department of Physics, Aristotle University of Thessaloniki \\ 54124, Thessaloniki, Greece
}

\begin{abstract}
Temperature dependences of magnetization of core-shell-type nanoparticles with non-magnetic core and ferromagnetic shell are obtained using Monte Carlo simulation. The influence of surface spin disorder of the ferromagnetic shell on overall shape of magnetization curve is analyzed. The magnetic state diagram (in shell thickness - surface anisotropy coordinates), separating collinear and non-collinear states, is determined.
\end{abstract}

PACS numbers: 75.75.+a, 75.40.Mg, 75.30.Et

\section{Introduction}

Magnetic nanostructures are of considerable interest because of their nanoscale size with high surface-to-volume atomic ratio and magnetic behaviour strongly different from bulk materials. They have various geometries and morphologies - from complex bulk-like compounds to low-dimensional systems such as self-assembled arrays of nanograins. As one of numerous examples with potential applications as magnetoresistive or drug delivery functional materials, the core-shell bimetallic magnetic nanoparticles have been recently prepared as arrays (AgCo [1]) or metal-polymer (CoRh [2]) systems. For such well-dispersed regular arrays of nanoparticles the effect of magnetic dipolar and interparticle interactions is expected to be negligible and magnetic properties closer to those of a single nanoparticle. The hypothesis of core-shell nanoparticles, consisting of non-metallic core surrounded by a ferromagnetic (FM) shell, was first proposed in Ref. [2]. Embedding of $\mathrm{CoPt}$ and $\mathrm{CoRh}$ in a polymer matrix allowed to study the magnetic properties of a single nanoscale grain and minimized the effects of nanograins coupling. Steady magnetic fields of $5 \mathrm{~T}$ and pulsed magnetic fields of $15 \mathrm{~T}$ were insufficient to saturate the magnetization of small $(\approx 1.8 \mathrm{~nm}) \mathrm{CoRh}$

*corresponding author; e-mail: et@et.pfi.lt 
nanograins. This effect was explained [3] by small size of the particles and reduced exchange-to-site anisotropy ratio $J / K$. Unsaturated magnetization at fields up to $5.5 \mathrm{~T}$ was also observed in AgCo bimetallic nanograins produced by a colloidal (wet) chemistry technique [1]. This result was attributed to unusual multiphase polycrystalline structure of the materials with non-collinear arrangement of magnetic moments and highly disordered magnetic surface layer. A very likely reason of magnetic spins resistance to saturation for high magnetic fields is large surface anisotropy which originates from surface-core strains or magnetostriction and usually keeps the orientation of surface magnetic moments perpendicular to the surface [4]. Here we study by the Monte Carlo method the effects of surface anisotropy in core-shell nanograin.

\section{Model}

We have chosen a sphere of radius $R=R_{\mathrm{A}}+R_{\mathrm{B}}$ composed of two parts: the sphere of a smaller radius $R_{\mathrm{A}}$ in a core, consisting of non-magnetic spins $\mathrm{A}$, embedded in a FM shell of strongly coupled spins B (located in sites of a simple cubic lattice) with thickness of the shell $R_{\mathrm{B}}$. The energy of such a system is written in terms of a classical nearest-neighbour exchange Heisenberg spins Hamiltonian with surface and bulk anisotropy terms included in a form

$$
H=-\sum_{i, j} J_{i j} \boldsymbol{S}_{i} \boldsymbol{S}_{j}-\sum_{i \in B_{V}} K_{V}\left(\boldsymbol{S}_{i} \cdot \boldsymbol{e}_{i}\right)^{2}-\sum_{i \in B_{S}} K_{S}\left(\boldsymbol{S}_{i} \cdot \boldsymbol{n}_{i}\right)^{2} .
$$

The first term describes the exchange spin coupling which is equal to zero in A part, $J_{\mathrm{B}}>0$ in $\mathrm{B}$ part, and $J_{\mathrm{AB}}>0$ at the core-shell interface. Only B spins are affected by bulk uniaxial ( $z$-axis) and surface anisotropies represented by anisotropy constants $K_{V}$ and $K_{S}$, respectively. The surface anisotropy (experienced by surface spins only) in accordance with experiments (see [3-6] and references therein) is directed normal to the surface of the sphere.

The calculation is performed by the Monte Carlo method with free boundary conditions using standard Metropolis algorithm. We start from random spin configuration at high temperature and proceed by lowering temperature into the FM phase. We calculate the magnetizations in A and B parts

$$
M_{\mathrm{A}, \mathrm{B}}=\left(\sum_{i=1}^{N_{\mathrm{A}, \mathrm{B}}} m_{i}\right) / N_{\mathrm{A}, \mathrm{B}},
$$

total magnetization

$$
M=\frac{N_{\mathrm{A}} M_{\mathrm{A}}+N_{\mathrm{B}} M_{\mathrm{B}}}{N_{\mathrm{A}}+N_{\mathrm{B}}}
$$

along each of the axes, root-mean square magnetization $M_{\mathrm{rms}}$, and magnetic susceptibility to determine the value of $\mathrm{FM}$ phase transition $T_{\mathrm{c}}^{\mathrm{B}}$. Usually $4 \times 10^{4} \mathrm{MCS} /$ spin were enough to achieve the equilibrium. Further, the tempera- 
ture, all interaction and anisotropy constants are normalized to $J_{\mathrm{B}}$ and expressed in temperature units, therefore $k_{\mathrm{B}}=1$.

\section{Results}

We use the term "nanograin" for spherical particles for which the effects of surface anisotropy, surface-to-bulk ratio and FM shell size are important. Size effects might be considered as negligible and magnetic properties closer to the properties of bulk material for the grains of $R>10$, if all the sphere is FM $\left(R_{\mathrm{A}}=0\right)$. However we are mostly interested in magnetic properties of nanograins at rather high values of $R_{\mathrm{A}}$ when FM shell is thin and affected by surface anisotropy. When the core is non-magnetic, the form of magnetization curve is determined by $T_{\mathrm{c}}^{\mathrm{B}} \sim J_{\mathrm{B}}$ and by the size of FM shell, $R-R_{\mathrm{A}}$. The decrease in the latter parameter leads to notable $2 \mathrm{D}$ effects and decrease in $T_{\mathrm{c}}^{\mathrm{B}}$.

Surface and bulk anisotropies affect only the spins of FM B part. The form of magnetization and its saturation value (mostly determined by $M_{\mathrm{B}}$ value) strongly depends on competition between surface anisotropy and exchange coupling $J_{\mathrm{B}}$. For nanograins $K_{S}>K_{V}$ and $K_{S}<1$ is expected, however even qualitatively it is quite complicated to determine this ratio e.g. for $\mathrm{AgCo}$ or CoRh. Here we take $K_{S}=0.2$ and vary $K_{V}$ in rather large limits.
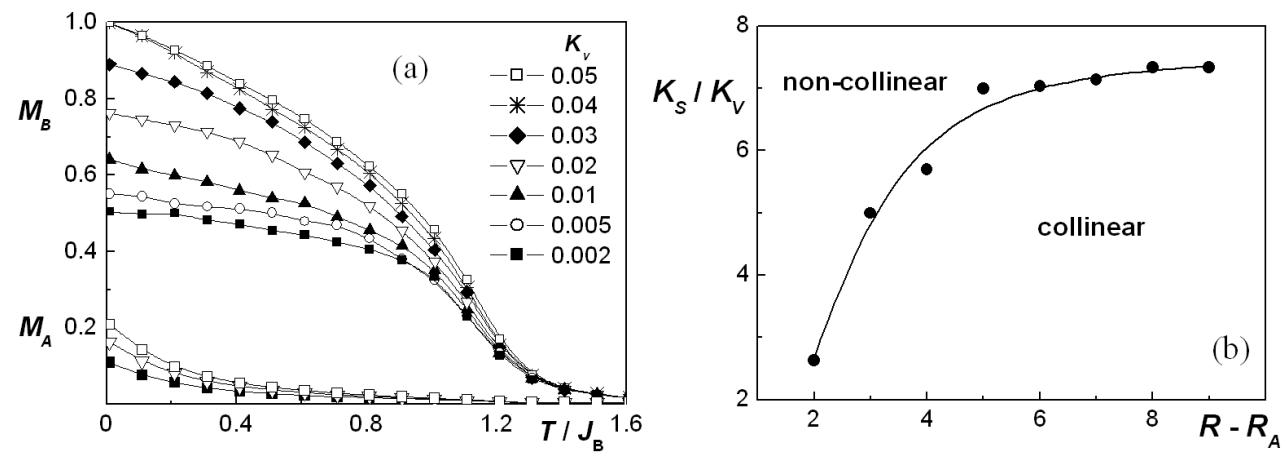

Fig. 1. (a) Temperature dependence of magnetizations $M_{\mathrm{A}}$ and $M_{\mathrm{B}}$ for $K_{V}$ ranging between 0.04 and 0.001 ; (b) state diagram between collinear and non-collinear states. For (a) and (b) $J_{\mathrm{AB}}=0.2$ and $K_{S}=0.2$.

For $K_{S}<1$ temperature dependence of magnetization (see Fig. 1a) demonstrates two thresholds. First, there is the threshold of $K_{S} / K_{V}$ ratio below which the ground state magnetization of the ferromagnetic shell is still collinear along the easy axis, $M_{\mathrm{B}}^{z}(T=0)=1$, and $M^{z}(0)=M_{\mathrm{rms}}(0)$ in spite of surface anisotropy. Only by exceeding the threshold value of $K_{S} / K_{V}, M_{\mathrm{B}}^{z}(0)$ starts to decrease and $M^{z}(0)<M_{\text {rms }}(0)$. This threshold obviously divides collinear (Fig. 2a) and non-collinear (Fig. 2b) magnetic states and reminds three different regions of magnetization (collinear, throttled, and hedgehog) obtained for different $K_{S} / K_{V}$ values 

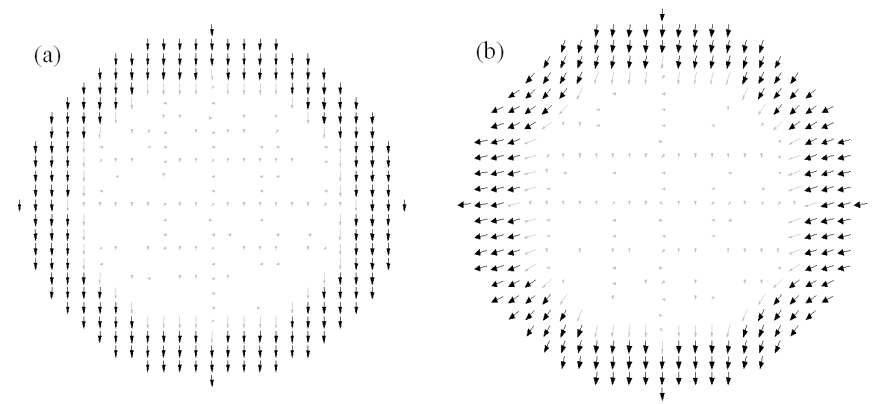

Fig. 2. Central section of $R=12$ nanosphere at the ground state for $J_{\mathrm{AB}}=0.2$, $K_{S}=0.2$ and $K_{V}=0.08$ (a) and $0.01(\mathrm{~b})$.

by numerical simulations of small $(R=6) \mathrm{FM}$ sphere [4]. The threshold obtained by us depends on a thickness of the FM shell. The state diagram (in $K_{S} / K_{V}$ and $R-R_{\mathrm{A}}$ coordinates), separating collinear and non-collinear states, is given in Fig. 1b. We have also noticed second threshold - starting from some $K_{S} / K_{V}$ value further decrease in $K_{V}$ has very little effect on $M_{\mathrm{B}}^{z}(0)$ (Fig. 1a). The magnetization curves for $K_{S} / K_{V}>40$ give the value of $M_{\mathrm{B}}^{z} \approx 0.55-0.5$, the state is partially throttled - the central spins are collinear along the easy axis and side spins are trying to accommodate themselves in accordance with requirement of normality to the surface (Fig. $2 \mathrm{~b}$ ). Hence, pure throttled phase with central spins holding an angle close to 45 degrees with the easy axis at $K_{S} / K_{V}=10^{2}-10^{3}$, is characteristic only of small $(R \approx 6$ or similar) spheres.

In summary, the effects of surface anisotropy on spin state of FM shell in bimetallic non-magnetic core - FM shell nanoparticles are studied by the Monte Carlo method when the magnitude of the surface anisotropy is less than FM exchange coupling in the shell. The surface-to-bulk anisotropy ratio, separating collinear and non-collinear states, is determined for different sizes of the shell.

\section{Acknowledgments}

The authors are grateful for EC project PRAMA for financial support.

\section{References}

[1] O. Crisan, M. Angelakeris, M. Noguès, Th. Kehagias, Ph. Komninou, E.N. Sobal, M. Giersig, N.K. Flevaris, J. Magn. Magn. Mater. 272-276S, E1253 (2004).

[2] D. Zitouin, M. Respaud, M-C. Fromen, M. J. Casanove, P. Lecante, C. Amiens, B. Chaudret, Phys. Rev. B 89, 037203 (2002).

[3] D.A. Dimitrov, G.M. Wysin, Phys. Rev. B 50, 3077 (1994); 51, 11947 (1995).

[4] Y. Labaye, O. Crisan, L. Berger, J.M. Greneche, J.M.D. Coey, J. Appl. Phys. 91, 8715 (2002).

[5] R.H. Kodama, A.E. Berkowitz, Phys. Rev. B 59, 6321 (1999).

[6] H. Kachkachi, M. Dimian, Phys. Rev. B 66, 174419 (2002). 